\title{
Evaluación del uso de alcohol por familias de la periferia de Guayaquil en Ecuador, por estudiantes de enfermería
}

\author{
Marìa Mieles Moreira ${ }^{1}$ \\ Sueli Aparecida Frari Galera²
}

\begin{abstract}
La presente investigación tiene como objetivo presentar el perfil socioeconómico y demográfico y las características del uso de alcohol de las familias evaluadas durante la cátedra de Enfermería Familiar y Comunitaria. Se trata de estudio de caso que utilizó como fuente de datos el Formulario de Valoración Familiar aplicado por estudiantes de la disciplina de Enfermería Familiar y Comunitaria. Las familias fueron seleccionadas por conveniencia, según los indicadores de riesgo: pobreza informada por las condiciones externas de la vivienda o identificadas por el servicio de salud. Se elaboró un formulario que tuvo como base el formulario usado por las estudiantes para recolectar la información de las familias; el cual contenía información sobre el perfil socioeconómico, características familiares y consumo de alcohol. En familias que informaron el uso de alcohol se aplicó el instrumento The Alcohol Users Identification Desorder Test (AUDIT). De las 128 familias evaluadas por las estudiantes encontramos 47 que informaron el uso de alcohol, las cuales fueron seleccionadas para responder el AUDIT. El promedio en la puntuación total obtenida fue de 10,66 puntos indicando Zona 2 de riesgo. Para este nivel está indicada intervención con consejo simple, que puede ser aplicado por los profesionales de atención primaria, principalmente enfermeros.
\end{abstract}

Descriptores: Alcoholismo; Composición Familiar, Atención Primaria de Salud.

\footnotetext{
${ }^{1}$ Enfermera, Escuela de Enfermería, Universidad de Guayaquil, Ecuador. E-mail: mamimo67@yahoo.com.mx.

2 Enfermeira, Doutor em Enfermagem. Professor Doutor, Escola de Enfermagem de Ribeirão Preto, Universidade de São Paulo, Centro Colaborador de la OMS para el Desarrollo de la Investigación en Enfermería, Brasil. E-mail: sugalera@eerp.usp.br.
}

Correspondencia:

Sueli Aparecida Frari Galera

Universidade de São Paulo. Escola de Enfermagem de Ribeirão Preto

Av. Bandeirantes, 3900

Bairro Monte Alegre

CEP: 14040-902 Ribeirão Preto, SP, Brasil

E-mail: sugalera@eerp.usp.br 


\title{
Avaliação do uso de álcool por famílias moradoras da periferia de Guayaquil, Equador, por estudantes de enfermagem
}

Este trabalho teve como objetivo apresentar o perfil sociodemográfico e o uso de álcool por famílias avaliadas em estágio da disciplina Enfermagem Familiar Comunitária. Trata-se de estudo de caso que utilizou como fonte de dados o Formulário de Avaliação Familiar aplicado por estudantes. As famílias foram selecionadas por conveniência segundo o indicador de risco pobreza, informada pelas condições externas das moradias ou identificadas pelo serviço de saúde. Elaborou-se um formulário para se obter as informações a partir do formulário aplicado pelas estudantes, o qual continha informações sobre o perfil socioeconômico, características familiares e consumo de álcool. Para as famílias que informaram o uso de álcool aplicou-se o The Alcohol Users Identification Desorder Test (AUDIT). Das 128 famílias avaliadas, 47 informaram a presença de consumo de álcool, as quais foram selecionadas para responderem AUDIT. A pontuação média obtida foi de 10.66 pontos indicando Zona 2 de risco, nível que é indicado para a aplicação de intervenção com aconselhamento simples que podem ser realizados pelos profissionais da atenção primária, principalmente enfermeiros.

Descritores: Alcoolismo; Características da Família; Atenção Primária à Saúde.

\section{Assessment of alcohol use in families of the suburbs of Guayaquil City, Ecuador, by nursing students}

\begin{abstract}
The aim of this study was to present the demographic and alcohol use profiles of families assessed by nursing students during the Community and Family Nursing Program. This is a case study using "The family assessment questionnaire" as its data source, which was applied by nursing students to assess family health conditions, during their practical training. Family selection was by convenience according to risk indicators and poverty, assessed from the outside condition of the dwelling or from public health services records of the area. A questionnaire correlated with the one that students applied was prepared to obtain data. This instrument obtained information about the socioeconomic profile, family environment and alcohol consumption. The Alcohol Use Disorder Identification Test (AUDIT) was applied to families that reported alcohol use. Of the 128 families evaluated by students we found 47 families who reported alcohol consumption, these families answered AUDIT, the average total score was 10.66 which equates to risk level 2. The suggested intervention for this level of risk is simple counseling that can be offered by nurses in the primary health care.
\end{abstract}

Descriptors: Alcoholism; Family Characteristics; Primary Health Care.

\section{Introducción}

El consumo de alcohol en las familias constituye una de las problemáticas sociales actuales de mayor trascendencia. Principalmente cuando se quiere hacer referencia a las condiciones de salud, y a los efectos que el alcoholismo ocasiona en la salud familiar; con el agravante del contexto de baja situación socioeconómica y de desarrollo donde viven algunos grupos de nuestros ciudadanos.

La familia, considerada como célula vital para la salud humana, es el contexto dentro del cual evoluciona la salud del individuo. Ella influye de manera significativa en las creencias de sus miembros, en sus actitudes y en sus comportamientos relativos a la salud y enfermedad. Los hábitos como alimentación, uso de alcohol y tabaco, 
practicar ejercicios físicos, la manera de lidiar con situaciones de estrés, se desarrollan dentro del contexto familiar(1).

Los principales factores para iniciar el consumo de alcohol y tabaco en el medio familiar, son el factor económico, la baja escolaridad, el padre bebedor $y$, el tipo de trabajo(2). Aproximadamente uno de cada cuatro niños, en las familias, está expuesto al abuso del alcohol o adicción ${ }^{(3)}$. Los hijos de padres alcohólicos tienen más probabilidades de empezar a beber durante su adolescencia y a desarrollar problemas relacionados al uso de alcohol. La forma en que los padres beben alcohol y sus actitudes favorables hacia la bebida están asociadas con el hecho de que sus hijos adolescentes comiencen a beber alcohol y continúen haciéndolo(4-5). El uso de alcohol por hermanos mayores puede influir en el uso de alcohol por menores de edad en la familia, particularmente por hermanos del mismo sexo(6).

Por otro lado, la familia también protege a sus miembros del uso nocivo del alcohol. Las investigaciones más recientes sugieren que hay menos probabilidades de que los niños beban alcohol si los padres forman parte de sus vidas y participan en sus actividades y cuando tanto los niños como los padres indican que tienen una relación estrecha(7). Los adolescentes beben menos y tienen menos problemas relacionados con el alcohol cuando los padres aplican una disciplina uniforme $y$ expresan sus expectativas claramente(8).

La enfermera de atención primaria busca conocer la población que vive en torno a los servicios básicos de salud; por esto, tiene una posición privilegiada para identificar familias donde el uso del alcohol puede ser perjudicial para la salud del grupo y proponer estrategias para promover acciones protectoras en relación al uso de alcohol(9).

La formación de estudiantes de enfermería en la Escuela de Enfermería de la Universidad de Guayaquil, está enfocada en las normas de Atención Primaria de la Salud. En el primer año, en la cátedra de Enfermería Familiar y Comunitaria, los estudiantes realizan visitas a domicilio y aplican el Formulario de Valoración Familiar desarrollado y estandarizado por los docentes de la disciplina basándose en el Modelo de Valoración Integral de Salud(10).

El Modelo de Valoración Integral de Salud se puede aplicar al individuo, familia y comunidad, consta de 11 ítems que son: manejo de salud, nutricional metabólico, eliminación, actividad y ejercicio, sueño y descanso, cognitivo perceptual, auto percepción, auto concepto, rol relaciones, adaptación y tolerancia al estrés, sexualidad y reproducción, valores y creencias.
La Escuela de Enfermería utiliza como campo de estudio práctico de la cátedra, "Enfermería Familiar y Comunitaria", los servicios de salud del sector suroeste, la Trinitaria, Guasmo Sur, Limonales, Martha de Roldós, Pancho Jácome y Bastión Popular. Barrios urbanos marginales de la ciudad de Guayaquil, donde existe más riesgo para la salud.

Las estudiantes de la Escuela de Enfermería reciben formación en la prevención comprensiva del uso de drogas como parte del currículo. De esta forma en la Cátedra de enfermería Familiar y Comunitaria se realizan evaluaciones por estándares funcionales, donde se incluye también el fenómeno de las drogas.

Para los estudios prácticos se seleccionan familias que habitan en sectores periféricos de la ciudad, que son sectores donde los índices de criminalidad y violencia son muy elevados. Se aplica el proceso de atención de enfermería en todas sus fases, que incluyen la valoración, diagnóstico, planificación e implementación, realizando programas de prevención con familias, escuelas y demás grupos comunitarios.

Considerando la participación, de una docente de la cátedra de Enfermería Familiar y Comunitaria, en el curso de formación en investigación sobre el fenómeno de alcohol y droga para la América Latina, promovido por la Comisión Interamericana para el Control de Drogas - CICAD en coordinación con la Escuela de Enfermería de Ribeirao Preto-Universidad de São Paulo, se decidió realizar un levantamiento de familias evaluadas durante la cátedra de Enfermería Familiar y Comunitaria que pueden estar en riesgo por causa del uso de alcohol.

\section{Objetivo}

El objetivo de este trabajo es presentar el perfil sociodemográfico y las características del uso del alcohol en las familias evaluadas durante el período de entrenamiento de la cátedra enfermería familiar comunitaria.

\section{Método}

Se trata de un estudio de caso que utilizó como fuente de datos el Formulario de Valoración Familiar aplicado por estudiantes en la cátedra de Enfermería Familiar y Comunitaria.

\section{Participantes}

Durante las prácticas de la cátedra Enfermería Familiar y Comunitaria, los estudiantes visitaron y evaluaron 128 familias que vivían en sectores periféricos de la ciudad de Guayaquil, distribuidas en 7 sectores: 
Guasmo Sur, Trinitaria, Limonales, Juan Montalvo, Bastión Popular, sector suroeste y Martha de Roldós.

Estas familias fueron seleccionadas según los indicadores de riesgo: pobreza detectada por las condiciones externas de la vivienda o identificadas por la unidad operativa.

\section{Instrumentos}

Se elaboró un formulario que contenía información sobre el perfil socioeconómico, características familiares y de consumo de alcohol. En familias que manifestaron consumir alcohol se aplicó el instrumento llamado The Alcohol Use Disorder Identification Test (AUDIT)(11) con el objetivo de determinar los niveles del riesgo de problemas relacionados con el alcohol. El AUDIT fue validado, a través de estudio comparativo conducido en seis países, Noruega, Australia, Kenia, Bulgaria, México e Estados Unidos ${ }^{(12)}$

\section{Procedimiento}

En el período de enero a marzo del 2007, las estudiantes del primer año en la cátedra de Enfermería Familiar y Comunitaria, visitaron las familias y realizaron la evaluación familiar utilizando el Formulario de Valoración Familiar. En las familias que manifestaron el consumo de alcohol se aplicó el AUDIT. Un miembro de la familia proporcionó todas las informaciones.

Antes de la recolección de la información, se explicó a los estudiantes que la evaluación de las familias serviría como fuente de datos para realizar la investigación. Se insistió en la veracidad y el llenado completo de los formularios de evaluación familiar para evitar errores en la obtención de datos, o la ausencia de los mismos. Además, se instruyó a las estudiantes para que al encontrar algún miembro de la familia que consumiese alcohol, se le aplicase el formulario AUDIT, para medir el nivel de riesgo, como se expresa en los resultados de este estudio. La recolección de los datos estuvo supervisada por la docente investigadora.

\section{Análisis de los datos}

Los formularios aplicados por las estudiantes fueron recolectados y los datos de interés para la investigación fueron recolectados a través de un formulario que se elaboró para este efecto. Se utilizó la frecuencia y el porcentaje.

\section{Aspectos éticos}

La investigación recibió el soporte y la autorización de la dirección de la escuela de Enfermería y del
Departamento de investigaciones, así como del comité provisional de Bioética de la Universidad de Guayaquil. La investigación no contó con la autorización de las personas que constituyen las familias evaluadas, ya que los datos fueran recolectados a partir del formulario utilizados por las estudiantes. Por tanto se trata de datos secundarios.

\section{Resultados y Discusión}

A seguir presentamos las características de las 128 familias que fueron evaluadas por los estudiantes de enfermería durante las prácticas en la cátedra de Enfermería Familiar y Comunitaria.

\section{Perfil socioeconómico}

\section{Procedencia de la familia}

La mayoría (73\%) de las familias provienen de la misma ciudad en que viven, en este caso la ciudad de Guayaquil. Por tanto se trata de familias urbanas ya acostumbradas al ambiente.

\section{Escolaridad de los padres}

Padres: $47 \%$ posee educación primaria, el $40 \%$ secundaria, el $13 \%$ superior y menos del $1 \%$ posee otro tipo de educación. Madres: 43\% posee educación primaria, el $46 \%$ secundaria y el $12 \%$ posee educación superior.

Situación laboral de los padres

El 50,4\% posee trabajo propio, el $48,8 \%$ trabajo ajeno, el 9,3\% tareas en casa, 1,6\% desempleado y el $3,9 \%$ otro tipo de trabajo.

Se debe considerar que esta variable recopila información del padre y madre en la familia al mismo tiempo, y que la mayoría de los trabajos propios son comercios menores los que realmente no le da solvencia económica y más aun ocasiona un grave problema en los ingresos familiares que son mínimos como lo indican los siguientes resultados:

El $22 \%$ de las familias poseen ingresos menores que $\$ 100,42 \%$ ingresos entre $\$ 101$ y $\$ 200,22 \%$ ingresos entre $\$ 201$ y $\$ 400$ y, el $14 \%$ ingresos mayores que $\$ 400$. El porcentaje que reciben las familias como salario es muy bajo, este no alcanza a cubrir las necesidades básicas. En muchas familias el dinero no alcanza a cubrir la canasta familiar que, en nuestro país (Ecuador) según el Instituto Ecuatoriano de Estadísticas y Censo es de $\$ 360$ (INEC -2006). 
Acceso al servicio de salud

El estudio revela que la mayoría $(52,7 \%)$ de estas familias acceden a unidades operativas del Ministerio de Salud Pública, 21,7\% reciben asistencia privada que en algunas ocaciones no están certificadas, regresando por atenciòn nuevamente a las unidades de salud del Estado.

\section{Características familiares}

Podemos resaltar que un gran porcentaje (48\%) de familias están conformadas por 3 o 4 miembros; y el $32 \%$ están poseen entre 5 y 7 miembros, siendos estos los grupos más relevantes.

El estudio demuestra que la mayoría (69\%) señala ser familias nucleares, el 20\% familia extendida, el $10 \%$ familia monoparentales (Madre) y menos de $1 \%$ familia monoparentales (Padre).

Edades de las figuras parentales: La mayoría $(71,1 \%)$ tiene edad superior a 35 años, siendo este el intervalo de mayor concentración.

Edades de los hijos: Las edades de los hijos se agrupan en las siguientes categorías: el $28,9 \%$ son menores de 6 años, escolares el 42,2\%, adolescentes $43 \%$ y adultos el $36,7 \%$. Básicamente la mayoría son escolares y adolescentes, edad fundamental en la adquisición de hábitos y valores para la prevención del consumo de alcohol.

Actividades en la familia: La principal actividad que realiza el $73,4 \%$ de las familias es ver TV, en segundo lugar con el 55,5\% está comer en familia, más del $40 \%$ tratan de estudios, problemas y planes personales.

La principal actividad que realizan en el tiempo libre $(68,8 \%)$ las madres es dialogar con los hijos, a continuación el 20,3\% realiza actividas recreacionales, mientras que la principal actividad que realizan el $49,2 \%$ de los padres es dialogar con los hijos, luego el 37,5\% realiza actividas recreacionales. Aproximadamente el $50 \%$ de las familias consultadas asegura tener una muy buena relación familiar, el $41 \%$ una buena relación y el $9 \%$ una relación regular.

\section{Consumo de alcohol}

De las 128 familias evaluadas por las estudiantes de primer año de enfermería, 47(37\%) informaron hacer uso de alcohol y respondieron el AUDIT. Investigaciones con el objetivo de identificar problemas relacionados con el uso del alcohol, en el contexto de la atención primaria, verifican que el $20 \%$ de los pacientes visitados presentó problemas con el alcohol en un cierto momento de sus vidas $^{(13-14)}$.
En 33 familias existen padres que consumen alcohol, y en el caso de los hijos 14 lo consumen. De los padres que consumen alcohol el 54,8\% tiene educación primaria, y las madres el $100 \%$ de las que consumen tienen educación primaria. El 79\% nunca ingiere alcohol frente a los hijos. Con relación a problemas derivados del consumo de alcohol: el 81,1\% de las familias informó que no tienen problemas, el 9,3\% tenía problemas conyugales y el 7,8\% tenía discusiones fuertes.

\section{Puntuación total AUDIT}

El promedio en la puntuación total obtenida fue de 10,66 puntos, indicando Zona 2 de riesgo. El 40\% está en la Zona 1 de nivel de riesgo, el $32 \%$ se encuentra en zona 2 y el $28 \%$ está distribuido en las zonas 3 y 4 . Las intervenciones preconizadas para los nivel de riesgo 1 y 2 son el consejo simple y la educación sobre alcohol. Los niveles 3 y 4 exigen una evaluación más cuidadosa para el diagnóstico de dependencia o tratamiento de las personas $^{(11)}$.

\section{Conclusiones}

En conclusión, el porcentaje de consumo de alcohol en las familias, evaluadas por las estudiantes de enfermería, según el formulario de Valoración Familiar por patrones funcionales, fue de $37 \%$. La enfermera de salud comunitaria tiene posición privilegiada para identificar precozmente los riesgos de la dependencia del alcohol y para promover acciones de salud que puedan prevenir el desarrollo de los casos de riesgo ${ }^{(15)}$. También tuvimos la oportunidad de identificar familias que tenían miembros con problemas de dependencia contribuyendo para un mejor diagnóstico y tratamiento; también, para ofrecer apoyo a las familias de dependientes, fortaleciendo al grupo en el sentido de mantenerse saludables o manejar problemas de dependencia con mejores resultados.

En base a los datos obtenidos podemos concluir que la enseñanza práctica de Enfermería Familiar y Comunitaria contribuye para la práctica de atención primaria de la salud, pues permite identificar en las familias factores de riesgo, así como factores protectores para el consumo de alcohol.

La introducción del AUDIT en el formulario también mostró ser útil, pues permite identificar las familias que están en riesgo y realizar acciones de salud que puedan contribuir al enfrentamiento de la dependencia del alcohol en las poblaciones periféricas o marginales de la ciudad. 
El pequeño número de la muestra y el proceso de selección de las familias que participaran en la investigación son las principales limitaciones del estudio. Por tanto, los resultados no nos permiten hacer generalizaciones.

\section{Agradecimientos}

Agradecemos a la Comisión Interamericana para el Control del Abuso de Drogas/CICAD de la Secretaria de Seguridad Multidimensional/SSM de la Organización de los Estados Americanos/OEA, la Secretaria Nacional de Políticas sobre Drogas/SENAD do Gabinete de Seguridad Institucional/Brasil, la Escuela de Enfermería de Ribeirao Preto de la Universidad de Sao Paulo y Centro Colaborador de la Organización Mundial de la Salud para el Desarrollo de la Investigación en Enfermería, la población representada en los estudios de investigación, bien como a las autoridades de las universidades representadas por los participantes del Programa En-Line de Especialización en Investigación sobre el Fenómeno de las Drogas PREINVEST, periodos 2005, 2006, 2007 y 2008.

\section{Referencia}

1. Duhamel F. La santé et la famille: une approche systémique en soins infirmiers. Montréal:Gaëtan Morin Editeur, 1995.

2. US Department of Health and Human Services. Substance Abuse and Mental Health Services Administration. SA MASA. Results from the 2005 National Survey on Drug Use and Health. Rockville (MD): Office of Applied Studies: National Findings; 2006.

3. Grant BF. Estimates of US children exposed to alcohol abuse and dependence in the family. Am J Public Health 2000; $90(1): 112-5$.

4. National Institute on Alcohol Abuse and Alcoholism. Youth Drinking: Risk Factors and Consequences. Alcohol Alert [internet]. 1997; (37). Available from: http://pubs.niaaa.nih. gov/publications/aa37.htm

5. Hawkins JD, Graham JW, Maguin E. Exploring the effects of age of alcohol use initiation and psychosocial risk factors on subsequent alcohol misuse. J Stud Alcohol 1997; 58(3):280-90.

6. Andrews JA, Hops H, Ary D. Parental influence on early adolescent substance use: Specific and nonspecific effects. J Early Adolesc 1993; 13(3):285-310.

7. Ary DV, Tildesley $E$, Hops H. The influence of parent, sibling, and peer modeling and attitudes on adolescent use of alcohol. Int J Addictions 1993; 28(9):853-80.
8. Resnick MD, Bearman PS, Blum RW. Protecting adolescents from harm: Findings from the National Longitudinal Study on Adolescent Health. J Am Med Assoc 1997; 278(10):823-32.

9. McGue M, Sharma A, Benson P. Parent and sibling influences on adolescent alcohol use and misuse: Evidence from a U.S. adoption cohort. J Studies Alcohol 1996; 57(1):8-18.

10. Cesar $C$, Suarez A. Promociòn de la Salud y Prevenciòn de la Enfermedad, Enfoque en Salud Familiar. Cap 8. Colombia:Jàurigue Paulina; 2004.

11. Babor TF, Fuente JR, Saunders J, Grant M. Audit: The alcoholic use disorders identification test: Guidelines for use in primary health care. Geneva: World Health Organization; 1989. 12. Saunders JB, Aasland OG, Babor TF, Fuente JR, Grant M. Development of the Alcohol Use Disorders Identification Test (AUDIT):WHO collaborative project on early detection of persons with harmful alcohol consumption. II. Addiction 1993; 88:791804.

13. Harwood GA. Alcohol abuse - screening in primary care. Nurse Practitioner 2005; 30(2):56-61.

14. Ockene JK, Wheeler E, Adams A. Provider training for patient-centered alcohol counseling in a primary care setting. Arch Int Med 1997; 157(20):2334-41.

15. Vargas D, Luis MAV. Alcohol, alcoholism and alcohol addicts: conceptions and attitudes of nurses from district basic health centers. Rev. Latino-Am. Enfermagem 2008; 16(spe):543-50. 